\title{
ANGULAR DISTRIBUTION OF PHOTOEMISSION FROM SURFACES OF AMORPHOUS SOLIDS
}

\author{
A. JABŁońSKI \\ Institute of Physical Chemistry, Polish Academy of Sciences \\ Kasprzaka 44/52, 01-224 Warszawa, Poland
}

As follows from the formalism of X-ray photoelectron spectroscopy, knowledge of the angular distribution of photoemission is crucial for certain applications of quantitative X-ray photoelectron spectroscopy analysis. In the present work, the experimental data on the relative angular distribution of photoemission from solid materials are reviewed and compared with theoretical predictions. Noticeable discrepancies are usually observed. It has been proved that major part of the observed discrepancies can be ascribed to elastic photoelectron scattering. The commonly used formalism, where the elastic collisions are neglected, may be of insufficient reliability for certain solids, or in certain experimental geometries. This formalism can be easily extended to account for elastic photoelectron collisions by introducing two correction factors, $Q \mathrm{x}$ and $\beta_{\text {eff }}$. The second parameter, called the effective asymmetry parameter, describes the observed decrease in anisotropy of photoemission. Determination of the correction factors requires a reliable theory describing elastic electron scattering in the solid. A need arises for accurate differential and total elastic electron scattering cross-sections pertinent to kinetic energies of considered photoelectrons or the Auger electrons. The increasingly important role of electron transport in surface analysis has stimulated an effort to construct a complete database containing the differential and total atomic elastic scattering cross-sections.

PACS numbers: 79.60.-i, 72.10.-d, 34.80.Bm

\section{Introduction}

Surfaces of polycrystalline and amorphous solids are frequently submitted to the X-ray photoelectron spectroscopy (XPS) studies, in particular to the quantitative analysis of the surface layer. Examples of such solids are polymers, supported catalysts, biomaterials, high- $T_{\mathrm{c}}$ superconductors, etc. These applications of XPS require knowledge of possibly accurate relations between the recorded signal intensity and the concentration of a given element. According to the commonly used formalism, the contribution to the signal, $\mathrm{d} I$, emitted in the layer of thickness $\mathrm{d} z$ at the depth $z$ is given by the following expression:

$$
\mathrm{d} I=T D I_{0} A \Delta \Omega N\left(\mathrm{~d} \sigma_{\mathrm{X}} / \mathrm{d} \Omega\right) \exp (-z / \lambda \cos \alpha) \mathrm{d} z,
$$


where $T$ is the analyser transmission function, $D$ is the detector efficiency, $I_{0}$ is the flux of incident $\mathrm{X}$-rays, $A$ is the analysed area, $\Delta \Omega$ is the solid acceptance angle of the analyser, $N$ is the atomic density, $\lambda$ is the inelastic mean free path of analysed photoelectrons, and $\alpha$ is the detection angle with respect to the surface normal. Parameter $\mathrm{d} \sigma_{\mathrm{X}} / \mathrm{d} \Omega$ denotes the differential photoelectric cross-section. For unpolarized radiation and random orientation of atorrs or molecules, this cross-section is described by

$$
\mathrm{d} \sigma_{\mathrm{X}} / \mathrm{d} \Omega=\sigma_{\mathrm{X}} W(\psi, \beta)=\sigma_{\mathrm{X}} \frac{1}{4 \pi}\left[1-\frac{\beta}{4}\left(3 \cos ^{2} \psi-1\right)\right],
$$

where $\sigma_{X}$ is the total photoelectric cross-section, $\psi$ is the angle between the direction of $\mathrm{X}$-rays and the direction of analysis, and $\beta$ is the so-called asymmetry parameter. Assurning that the analysed area increases with detection angle $\alpha$ according to $A=A_{0} / \cos \alpha$, where $A_{0}$ is the area at normal direction of analysis, we obtain the following expression on integration of $\mathrm{Eq}$. (1):

$$
I=T D I_{0} A_{0} \Delta \Omega N \lambda\left(\mathrm{d} \sigma_{\mathrm{X}} / \mathrm{d} \Omega\right) .
$$

Extensive derivation of the above formalisrn was published by Fadley et al. [1]. As follows from their considerations, Eqs. (1) and (2) involve the following assumptions:

1. The X-ray refraction and reflection are neglected.

2. The X-ray attenuation within the analysed volume is negligible.

3. Elastic scattering of photoelectrons on atoms constituting the solid has insignificant effect on the recorded photoelectron intensity.

Fadley et al. [1] have already mentioned that the last assumption may not always be valid. This problem was frequently addressed in more recent publications [2-5]. These reports indicated that the neglecting of the elastic photoelectron collisions in the formalism of XPS can affect the theoretically predicted characteristics e.g. intensity, thickness analysed, etc. The common formalism can lead to considerable errors in certain experimental geometries. In the present work, stress is put mainly on angular dependencies describing photoelectrons leaving the solid. Knowledge of this distribution is of crucial importance in calculations associated with quantitative applications of XPS. As an example, the applications of angle-resolved XPS (ARXPS) are based on measurements of angular distribution of photoelectrons. Experimental intensities are eventually transformed into composition profiles in near-surface regions. Obviously, the corresponding formalism should be possibly realistic to provide meaningful results.

\section{Theory}

Example of the photoelectron trajectory in a solid is outlined in Fig. 1. Several photoelectron elastic collisions can occur affecting drastically the original direction of ernission. In effect, as indicated in Fig. 1, the photoelectron entering the analyser at an angle $\alpha$ can pass different distance in the solid as predicted by the common formalism. The probability of inelastic collision depends on the total distance travelled in the solid, and thus the monitored intensity cari also be different than the intensity predicted from a formalism neglecting the elastic 


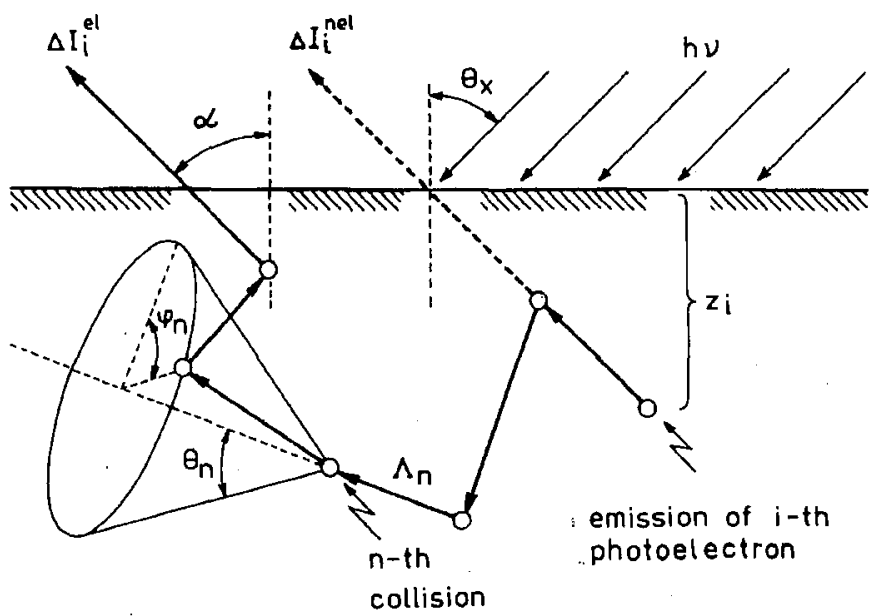

Fig. 1. Example of the photoelectron trajectory in the solid. $\Delta I_{i}^{\text {nel }}$ and $\Delta I_{i}^{\text {el }}$ denote the contributions to photoelectron current due to $i$-th trajectory neglecting or assuming presence of elastic collisions, respectively.

photoelectron collisions. Thus, we can expect that the angular distribution of photoemission from isolated atoms or molecules constituting the solid is different than the angular distribution of photoemission from solid.

The Monte Carlo method was frequently used for simulation of the electron transport in solids (Ref. [2] and references contained therein). The colresponding algorithm creates a number of trajectories similar to trajectory shown in Fig. 1. The following assumptions are usually made:

1. Angular distribution of created photoelectrons follows Eq. (1).

2. The trajectory is composed of linear steps, $\Lambda$, described by the exponential distribution.

3. The scattering event is defined by two angles, azimuthal, $\varphi$, and polar, $\theta$. The azimuthal angles are distributed uniformly between 0 and $2 \pi$. The distribution of polar scattering angles is related to the elastic scattering cross-sections:

$$
H(\theta)=\frac{\mathrm{d} \sigma / \mathrm{d} \theta}{\sigma_{\mathrm{t}}}=\frac{(\mathrm{d} \Omega / \mathrm{d} \theta)(\mathrm{d} \sigma / \mathrm{d} \Omega)}{\sigma_{\mathrm{t}}}=\frac{2 \pi \sin \theta(\mathrm{d} \sigma / \mathrm{d} \Omega)}{\sigma_{\mathrm{t}}},
$$

where $\mathrm{d} \sigma / \mathrm{d} \Omega$ is the differential elastic scattering cross-section, and $\sigma_{\mathrm{t}}$ is the total elastic scattering cross-section. A particular trajectory is followed until photoelectron leaves the solid or until the total trajectory length becomes excessively large (and the probability that the inelastic collisions do not occur becomes negligibly small). The contribution, $\Delta I_{i}^{\mathrm{el}}$, to the monitored current $I$ is calculated according to the following rule:

$$
\Delta I_{i}^{\mathrm{el}}=\left\{\begin{array}{cc}
\exp \left(-x_{i} / \lambda\right) & \begin{array}{c}
\text { if electron leaves the solid within } \\
\text { the solid angle of the analyser }
\end{array} \\
0 & \text { in all other cases }
\end{array}\right.
$$

where $x_{i}$ is the total trajectory length, and $\lambda$ is the inelastic mean free path (IMFP). 
The photoelectron intensity is estimated from

$$
I^{\mathrm{el}}=\lim _{n \rightarrow \infty} \frac{1}{n} \sum_{i=1}^{n} \Delta I_{i}^{\mathrm{el}},
$$

where $n$ is the number of trajectories. The Monte Carlo calculations require knowledge of elastic scattering cross-sections, $\mathrm{d} \sigma / \mathrm{d} \Omega$. Extensive tables containing these data are available in the literature [6-11]. However, their application in Monte Carlo simulations is limited since the cross-sections are available for selected elements and energies. The cross-sections for particular element and energy, equal to the kinetic energy of photoelectrons, should be determined from an algorithm implementing the so-called partial wave expansion method (PWEM). Numerous such algorithms were described in the literature [12-15]. As an example, a brief description of the relativistic PWEM algorithm is given below.

According to Lin et al. [13] and Bunyan and Schonfelder [14], the Dirac equation describing the scattering event on a spherical potential $V(r)$ can be transformed into the first order differential equation

$$
\begin{aligned}
& \frac{\mathrm{d} \Phi_{l}^{ \pm}(r)}{\mathrm{d} r}=\frac{k^{ \pm}}{r} \sin \left[2 \Phi_{l}^{ \pm}(r)\right]+[W-V(r)]-\cos \left[2 \Phi_{l}^{ \pm}(r)\right], \\
& l=0,1,2, \ldots
\end{aligned}
$$

where $W$ is the total electron energy, $k^{-}=-l-1$ for the "spin-up case" and $k^{+}=l$ for the "spin-down case". The system of units is such that energy is measured in units of $m_{0} c^{2}$ and the distance in units of $h / m_{0} c$. This equation describes the functions $\Phi_{l}^{ \pm}\left(r^{*}\right)$ which are related to the phase shiftis $\delta_{l}^{ \pm}$:

$$
\delta_{l}^{ \pm}=\lim _{r \rightarrow \infty}\left(\tan ^{-1} \frac{A}{B}\right),
$$

where

$$
\begin{aligned}
& A=K j_{l+1}(K r)-j_{l}(K r)\left[(W+1) \tan \Phi_{l}^{ \pm}(r)+\left(1+l+k^{ \pm}\right) / r\right], \\
& B=K n_{l+1}(K r)-n_{l}(K r)\left[(W+1) \tan \Phi_{l}^{ \pm}(r)+\left(1+l+k^{ \pm}\right) / r\right],
\end{aligned}
$$

$K^{2}=W^{2}-1$, and $j_{l}(x)$ and $n_{l}(x)$ are the spherical Bessel functions. The initial values of the functions $\Phi_{l}^{ \pm}(r)$ are calculated from the series expansions derived by Bunyan and Schonfelder [14]. On calculating the series of phase shifts $\delta_{0}^{ \pm}, \delta_{1}^{ \pm}, \delta_{2}^{ \pm}, \ldots$ the differential elastic scattering cross-sections are determined from the known expressions

$$
\mathrm{d} \sigma / \mathrm{d} \Omega=|f(\theta)|^{2}+|g(\theta)|^{2},
$$

where

$$
\begin{aligned}
& f(\theta)=-\frac{1}{2 \mathrm{i} K} \sum_{l}\left\{(l+1)\left[\exp \left(2 \mathrm{i} \delta_{l}^{+}\right)-1\right]+l\left[\exp \left(2 \mathrm{i} \delta_{l}^{-}\right)-1\right]\right\} P_{l}(\cos \theta), \\
& g(\theta)=\frac{1}{2 \mathrm{i} K} \sum_{l}\left[\exp \left(2 \mathrm{i} \delta_{l}^{-}\right)-\exp \left(2 i \delta_{l}^{+}\right)\right] P_{l}^{1}(\cos \theta)
\end{aligned}
$$

$P_{l}(\cos \theta)$ are the Legendre polynomials, and

$$
P_{l}^{1}(z)=\left(1-z^{2}\right)^{1 / 2} \frac{\mathrm{d} P_{l}(z)}{\mathrm{d} z} .
$$


The algorithm implementing Eqs. (4) $\div$ (6) for calculations of elastic scattering cross-sections is rather involved. Thus, it is not practical to use the corresponding software for fast reference, since calculations of cross-sections usually require a considerable computational effort. An effective solution to this problem is a creation of a computer controlled database providing the elastic scattering cross-sections for all elements and for a wide energy range. Such database has been recently reported in the literature [16]. Examples of the relativistic and nonrelativistic elastic scattering cross-sections are shown in Fig. 2.
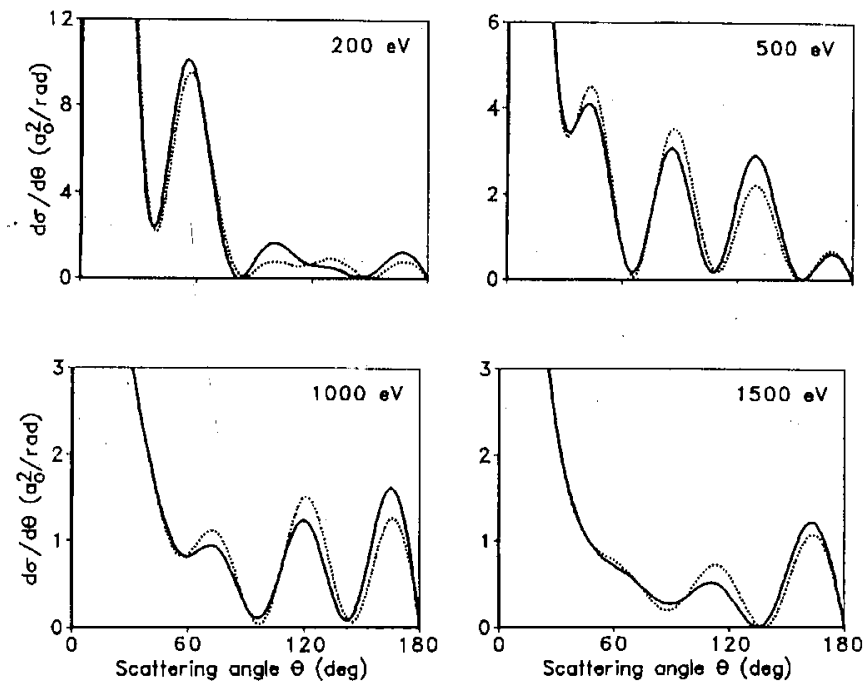

Fig. 2. Differential elastic scattering cross-sections calculated at different kinetic energies for gold. Solid line: nonrelativistic calculations; dotted line: relativistic calculations.

\section{Anisotropy of photoemission}

The theoretical studies of the angular distribution of photoemission from amorphous solids were initiated in 1979 by Baschenko and Nefedov [17]. The theory developed by these authors predicts a decay of anisotropy due to multiple elastic photoelectron collisions. This effect was studied in more detail in later reports $[2-5,18-20]$. A marked decrease in the anisotropy was found in all reported cases. Figure 3 shows comparison of intensity of $\mathrm{Au} 4 \mathrm{~s}$ photoelectrons calculated from Eq. (2) with results of the Monte Carlo calculations. These calculations are made for different XPS configurations described by angles $\alpha$ and $\theta_{\mathrm{X}}$ (Fig. 1). As one can see, the decrease in anisotropy does not depend critically on the detection angle $\alpha$.

As follows from Eq. (2), the anisotropy of photoemission from atoms and molecules is defined by the value of the asymmetry parameter. This is illustrated in Fig. 4. The isotropic photoernission, as shown in this plot, corresponds to the value of $\beta$ equal to 0 . Extensive theoretical data on the values of $\beta$ calculated for different subshells and different energies of radiation are available in the literature 


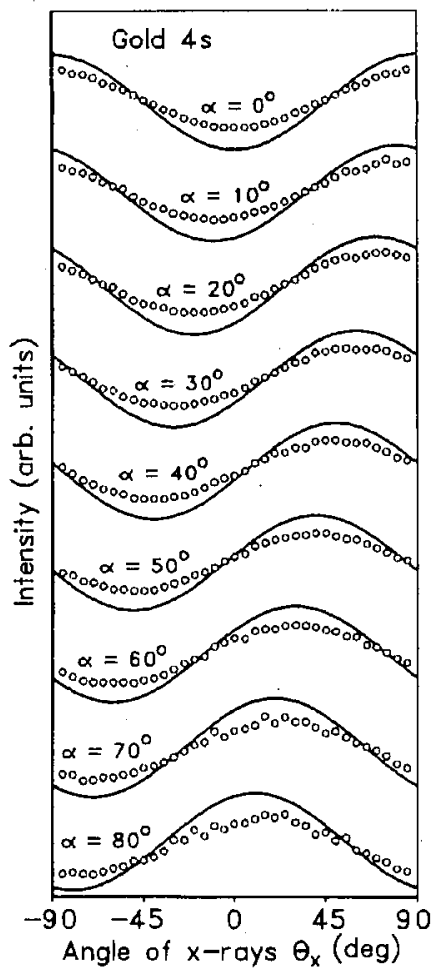

Fig. 3. Dependence of the photoelectron intensity on the detection angle, $\alpha$, and the incidence angle of X-rays, $\theta_{\mathrm{X}}$. Solid line: distribution of photoemission from isolated atoms (Eq. (2)); circles: Monte Carlo calculations.

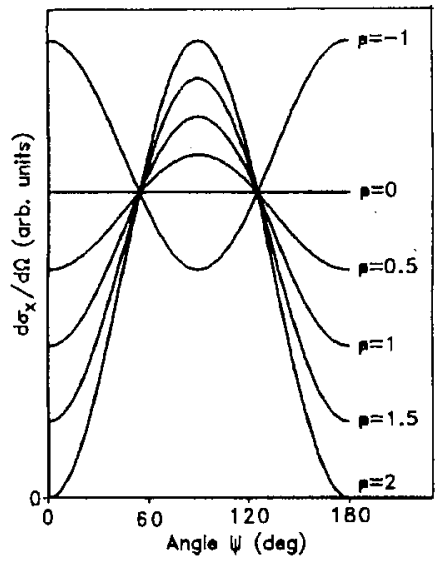

Fig. 4. Differential photoelectric cross-section, $\mathrm{d} \sigma_{\mathrm{X}} / \mathrm{d} \Omega$, for isolated atoms as a function of the angle $\psi$ and the asymmetry parameter, $\beta$. 
[21-24]. These values vary in the range from -1 to 2 . In considerable majority of cases, these values are positive. In several reports, the theoretical values of $\beta$ were compared with the experimental values obtained for different gaseous species [25-27]. A reasonably good agreement was found.

Within the common XPS formalism, as shown in the previous section, the angular distribution of photoemission from solid surfaces is assumed to be identical with the photoemission from isolated atoms or molecules. Vulli [28] seems to be the first to show experimentally that the angular distribution of photoemission from selected solids differs from the theory prediction. He has found that the measured anisotropy of photoemission from $s$ levels (i.e. of highest anisotropy) is somewhat less pronounced than the prediction for isolated atoms. However, the experimental values of $\beta$ for other subshells were found to be larger than the theoretical values. Such effect is unexpected since the interactions of photoelectrons with the solid should decrease the original anisotropy of emission. Thus, one can question the reliability of the above experiment. In 1984, the problem of angular distribution of photoemission from polycrystalline solid was addressed by Baschenko et al. [18]. These authors proposed a unique experimental arrangement which makes possible a relatively accurate measurements of the anisotropy of photoemission. The sample was a thin aluminium foil (about $5 \mu \mathrm{m}$ ) irradiated on one side with $\mathrm{X}$-rays. The foil of such thickness is transparent for X-rays and the photoemission can be observed on the other side of the foil. The described arrangernent enables unrestricted positioning of the movable analyser. Second modification, increasing the reliability of results, consists in measurements of the ratio of peak intensities instead of the intensity of a given peak. This way, the influence of the analysed area on intensity is cancelled. Baschenko et al. [18] have found that the ratio of the $\mathrm{Al} 2 s$ intensity to the $\mathrm{Al} 2 p$ intensity is markedly different from zero in the direction of X-rays. The asymmetry parameter for Al $2 s$ photoelectrons is equal to 2 and, as follows from Eq. (2), no photoelectron current should be observed in such XPS configuration. The intensity observed experimentally was ascribed by Baschenko et al. [18] entirely to elastic photoelectron collisions. This hypothesis was well supported by the Monte Carlo calculation using the published earlier algorithrr [17].

Similar experiments, with much larger number of data points, were repeated by Zemek and Jabłonski [19] and Jabłoński and Zemek [20]. In these studies, it has been found that the decay in anisotropy observed experimentally is even larger than the anisotropy resulting from Monte Carlo calculations. This is shown in Fig. 5a and b. Except for the elastic photoelectron interactions, the observed decay in anisotropy may be also caused by other factors. The finite solid acceptance angle of the analyser may distort the measured angular distribution of photoemission leading to apparent "flattening" of anisotropy. Such effect has been proved theoretically by Jabłonski et al. [29]. However, it has been found that the corresponding decrease in anisotropy is not pronounced for the acceptance angles usually used in XPS. This problem has also been approached experimentally by Jabłoński and Zemek [20]. Figure 5b shows the intensity ratios measured within two different acceptance angles: $\pm 4.1^{\circ}$ and $\pm 1.4^{\circ}$. As one can see, no marked difference can be observed. Angular distribution of photoemission can also be affected 

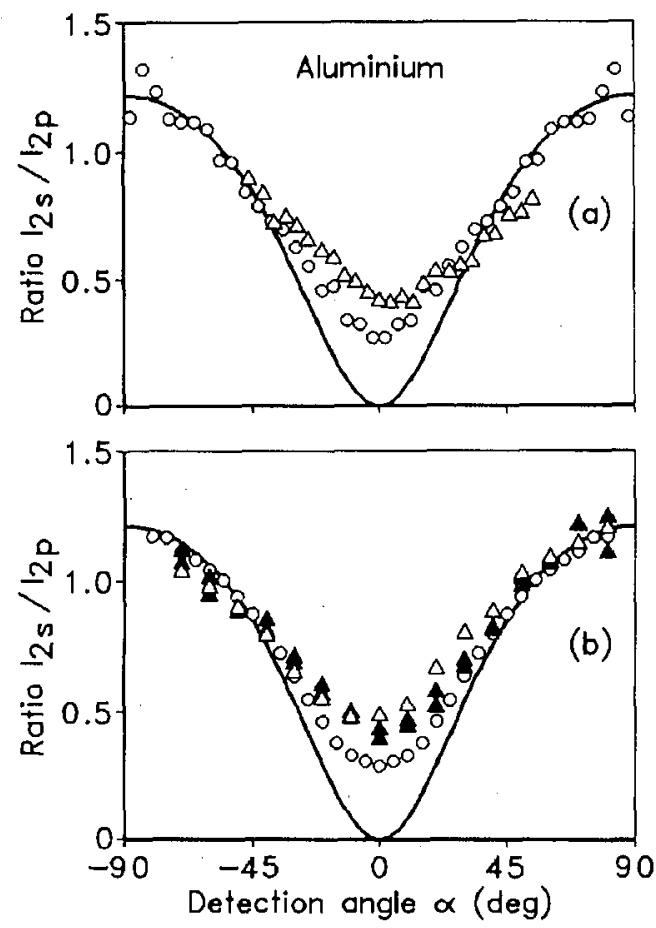

Fig. 5. The ratio of photoelectron intensities, $I_{2 s} / I_{2 p}$, for aluminium as a function of the detection angle. Solid line: common formalism based on Eq. (3); circles: Monte Carlo calculations; open triangles: experimental data collected with the acceptance angle $\pm 4.1^{\circ}$; solid triangles: experimental data collected with the acceptance angle $\pm 1.4^{\circ}$. (a) Figure taken from Ref. [19], (b) figure taken from Ref. [20].

by the surface roughness. Probably, this effect is responsible, to a large degree, for the observed difference between experimental ratios and the ratios resulting from the Monte Carlo calculations.

Jabłoński and Zernek [20] proposed a modification of the described above experimental geometry which makes possible the measurements of the angular distribution of photoemission from any material. This geometry is outlined in Fig. 6. The aluminium foil plays a role of a support. The studied material is deposited on one side of the foil. The thickness of the overlayer should be larger than the escape depth of photoelectrons, i.e. $10 \div 20 \hat{\AA}$. This is easily controlled during deposition by monitoring the photoelectron peaks from the support until they disappear. Overlayers of such thickness are also transparent for the exciting X-rays. Figure 7 shows the intensity ratios measured for gold overlayer. Similar effects as for aluminium are observed. Experimental ratios deviate considerably from the ratios resulting from the common formalism. The ratios obtained from the Monte Carlo calculations are relatively close to the experiment, although the difference is still noticeable. 


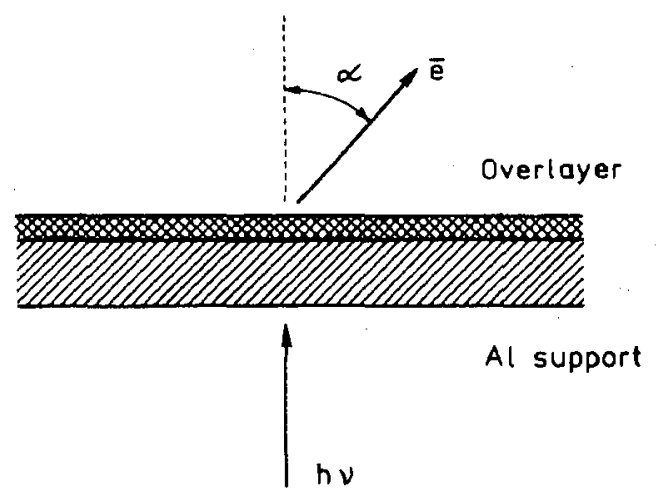

Fig. 6. Experimental geometry for measurements of the angular distribution of photoemission.
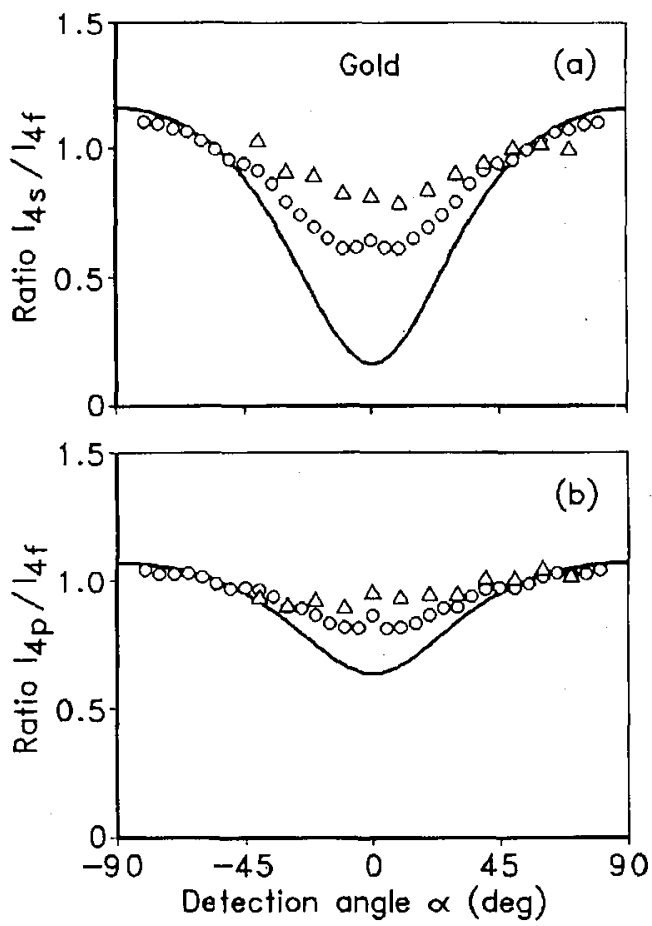

Fig. 7. The ratios of photoelectron intensities for gold as a function of the detection angle (taken from Ref. [20]). Notation as in Fig. 5. (a) The ratio $I_{4 s} / I_{4 f}$, (b) the ratio $I_{4 p} / I_{4 f}$

\section{Corrected formalism}

To facilitate calculations associated with quantitative applications of XPS, simple expressions approximating the Monte Carlo results were proposed in the 
literature $[2,5,20,30,31]$. Ebel et al. proposed to use the effective asymmetry parameter, $\beta_{\text {eff }}$, instead of the parameter $\beta$ to account for the decrease in anisotropy [30]. Since the decrease in anisotropy depends on the scattering properties of atoms constituting the solid, an attempt was made to introduce the atomic number dependence of $\beta_{\text {eff }}$ :

$$
\beta_{\mathrm{eff}}=0.92 k \beta,
$$

where

$$
k=1.0688-0.0235 Z+0.000188 Z^{2} .
$$

It has been found in later reports that the above approximation is not sufficiently accurate. Better fit was obtained with two adjustable parameters, $Q \mathrm{x}$ and $\beta_{\text {eff }}$ $[2,5,20,31]$ :

$$
\begin{aligned}
& \left(\mathrm{d} \sigma_{\mathrm{X}} / \mathrm{d} \Omega\right)_{\mathrm{el}}=\sigma_{\mathrm{X}} \frac{1}{4 \pi} Q_{\mathrm{X}} W\left(\psi, \beta_{\mathrm{eff}}\right) \\
& \quad=\sigma_{\mathrm{X}} \frac{1}{4 \pi} Q_{\mathrm{X}}\left[1-\frac{\beta_{\mathrm{eff}}}{4}\left(3 \cos ^{2} \psi-1\right)\right] .
\end{aligned}
$$

Replacement of the photoelectric cross-section, $\mathrm{d} \sigma_{\mathrm{X}} / \mathrm{d} \Omega$, in Eqs. (1) and (3) by Eq. (7) leads to formalism which is rnore realistic than the common formalism of XPS. Unfortunately, only limited data on the parameters $\beta_{\mathrm{eff}}$ and $Q_{\mathrm{X}}$ are available in the literature. They refer to selected XPS configurations: (i) with the detection angle equal to zero $[2,5,30,31]$, or (ii) with incidence angle of $\mathrm{X}$-rays equal to zero [20]. Extensive calculations are presently performed to provide the values of the parameters $\beta_{\mathrm{eff}}$ and $Q_{\mathrm{X}}$ for nurnerous configurations [32].

Let us consider modifications of the formalism associated with typical quantitative applications of quantitative XPS. Frequent application of XPS is the quantitative analysis of solid surfaces. Suppose that the measurement of a given peak intensity is done for a studied sample and for a certain standard (usually a pure element). According to a common formalism (Eq. (3)) we obtain

$$
\frac{I}{I^{\text {std }}}=\left(\frac{M}{M^{\text {std }}}\right)\left(\frac{\lambda}{\lambda^{\text {std }}}\right) x
$$

where $M$ is the total atomic density, $x$ is the atom fraction of a given element $(N=M x)$, and the superscript "std" refers to the standard. The modified formalism (Eqs. (3) and (7)) leads to the following expression:

$$
\frac{I}{I^{\text {std }}}=\left(\frac{M}{M^{\text {std }}}\right)\left(\frac{\lambda}{\lambda^{\text {std }}}\right)\left(\frac{Q \times}{} \frac{Q_{\mathrm{X}} W\left(\psi, \beta_{\mathrm{eff}}\right)}{Q_{\mathrm{X}}^{\text {std }} W\left(\psi, \beta_{\text {eff }}^{\text {std }}\right)}\right) x .
$$

Thus, additional factor appears which takes into account the difference between anisotropies of photoemission from the sample and the standard. In general, Eqs. (8) and (9) may provide different concentrations $x$, especially when the scattering properties of the surface region of the sample and the standard differ considerably, i.e. if $Q \mathrm{X} \neq Q_{\mathrm{X}}^{\text {std }}$ and $\beta_{\text {eff }} \neq \beta_{\text {eff }}^{\text {std }}$.

Another frequently used rrethod of quantitative analysis involves the so-called relative sensitivity factors, $\alpha$, defined as the proportionality factor between the peak intensity and the concentration

$$
I=\alpha x .
$$


From Eq. (3) we get

$$
\alpha=C S(E)(\mathrm{d} \sigma / \mathrm{d} \Omega) \lambda
$$

where $C=M \Delta \Omega I_{0}$ is a constant independent of the photoelectron line, $S(E)$ is the spectrometer function. For a given spectrometer, the relative sensitivity factors are determined experimentally or theoretically. The latter case is realized in procedures proposed by Ebel et al. [33] and Hanke et al. [34]. Obviously, Eq. (7) is more suitable to use in these procedures than $\mathrm{Eq}$. (2) for calculating the differential photoelectric cross-section.

Frequent quantitative application of XPS is the estimation of the overlayer thickness [35, 36]. Jabłoński et al. [31] published extensive analysis of variation of the emission anisotropy in systems with overlayers. It has been found that the correction factors $\beta_{\mathrm{eff}}$ and $Q_{\mathrm{x}}$ strongly depend on the overlayer thickness. Simple expressions describing this dependence were proposed. Thus, the above examples show that quantitative XPS requires the efficient methods providing the correction parameters $Q_{\mathrm{X}}$ and $\beta_{\mathrm{eff}}$ for complex systerns and for different XPS configurations.

\section{Conclusions}

The experimental data and the theoretical models of photoelectron transport in solids indicate that the initial anisotropy of photoemission is affected by the elastic interactions within the surface region of a solid. In effect, the angular distribution of photoemission from solid surface is different than the angular distribution predicted for isolated atoms or molecules. This phenomenon may be significant and should be accounted for in the formalism of quantitative XPS. Two correcting parameters, $Q_{\mathrm{X}}$ and $\beta_{\mathrm{eff}}$, describe the actual anisotropy of photoemission with sufficient accuracy. However, much work is still necessary to propose simple and effective methods providing these parameters for routine quantitative applications of XPS.

\section{References}

[1] C.S. Fadley, R..J. Baird, W. Siekhaus, T. Novakov, S.A. L. Bergström, J. Electron Spectrosc. Relat. Phenom. 4, 93 (1974).

[2] A. Jabłoúski, Surf. Interface Anal. 14, 659 (1989).

[3] W.S.M. Werner, I.S. Tilinin, Appl. Surf. Sci. 70/71, 29 (1993).

[4] I.S. Tilinin, W.S.M. Werner, Surf. Sci. 290, 119 (1993).

[5] A. Jabłoński, C.J. Powell, Surf. Interface Anal. 20, 771 (1993).

[6] M. Fink, A.C. Yates, At. Data Nucl. Data Tables 1, 385 (1970).

[7] M. Fink, J. Ingram, At. Data Nucl. Data Tables 4, 129 (1972).

[8] D. Gregory, M. Fink, At. Data Nucl. Data Tables 14, 39 (1974).

[9] M.E. Riley, C.J. McCallum, F. Biggs, At. Data Nucl. Data Tables 15, 443 (1975).

[10] L. Reimer, B. Lödding, Scanning 6, 128 (1984).

[11] Z. Czyzewski, D.O. MacCallum, A. Romig, D.C. Joy, J. Appl. Phys. 68, 3066 (1990). 
[12] F. Calogero, Variable Phase Approach to Potential Scattering, Academic Press, New York 1967.

[13] S.-R. Lin, N. Sherman, J.K. Percus, Nucl. Phys. 45, 492 (1963).

[14] P.J. Bunyan, J.L. Schonfelder, Proc. Phys. Soc. 85, 455 (1965).

[15] A. Jabłoński, Phys. Rev. B 43, 754.6 (1991).

[16] A. Jabłoński, S. Tougaard, Surf. Interface Anal., in press.

[17] O.A. Baschenko, V.I. Nefedov, J. Electron Spectrosc. Relat. Phenom. 17, 405 (1979).

[18] O.A. Baschenko, G.V. Machavariani, V.I. Nefedov, J. Electron Spectrosc. Rel 